\title{
Learning a Local Feature Descriptor for 3D LiDAR Scans
}

\author{
Ayush Dewan
}

Tim Caselitz

\begin{abstract}
Robust data association is necessary for virtually every SLAM system and finding corresponding points is typically a preprocessing step for scan alignment algorithms. Traditionally, handcrafted feature descriptors were used for these problems but recently learned descriptors have been shown to perform more robustly. In this work, we propose a local feature descriptor for 3D LiDAR scans. The descriptor is learned using a Convolutional Neural Network (CNN). Our proposed architecture consists of a Siamese network for learning a feature descriptor and a metric learning network for matching the descriptors. We also present a method for estimating local surface patches and obtaining ground-truth correspondences. In extensive experiments, we compare our learned feature descriptor with existing 3D local descriptors and report highly competitive results for multiple experiments in terms of matching accuracy and computation time.
\end{abstract}

\section{INTRODUCTION}

For many robotics tasks, it is required to have robust data association in order to match same parts of the scene under different conditions. Estimating data association is always an important step in SLAM systems [16] and different methods for lifelong visual localization [13] rely on finding corresponding points between scenes captured in different seasons. Furthermore, knowing corresponding points is also a requirement for different scan alignment methods [4]. In this work, we propose a local feature descriptor for 3D LiDAR data. Our proposed Convolutional Neural Network (CNN) architecture learns the feature descriptor and the metric for matching the descriptor in a unified way. Additionally, we propose a method for generating local surface patches and discuss an approach for obtaining ground-truth correspondences.

The majority of existing feature descriptors for 3D data are handcrafted [2] and rely either on quantifying surface normals or curvature around keypoints. In contrast to these methods, we do not try to explicitly extract geometric information but instead use raw scan data. In this work, we focus on using LiDAR scans and learn descriptors for two channel surface patches encoding local shape and surface reflectance values.

Recently, various CNN-based methods have been proposed for learning feature descriptors for image patches [7, 20, 3] and dense 3D surface patches [21]. All of these methods include a two or multi-stream Siamese network for learning a feature descriptor which is either discriminative in a predefined [20, 3, 21] or in a learned metric [7]. Our proposed architecture consists of a two-stream Siamese network for learning a descriptor, followed by a network for learning

All authors are with the Department of Computer Science at the University of Freiburg, Germany.

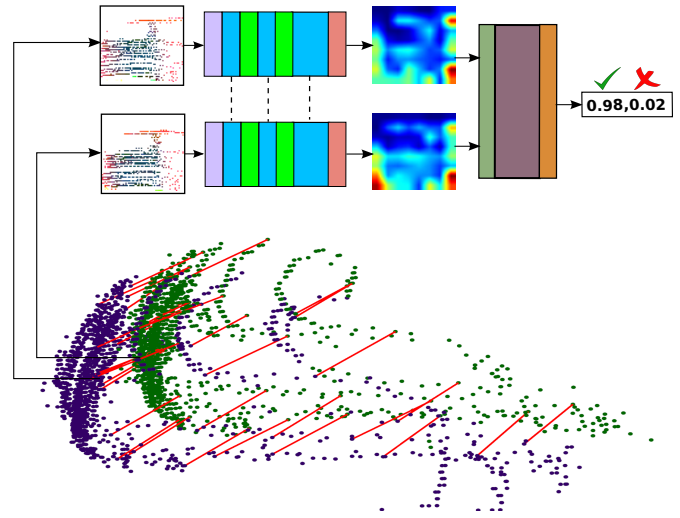

Fig. 1: An illustration of keypoint matching using our learned feature descriptor. Surface patches around the keypoints are passed through the feature learning network to estimate feature descriptors. These descriptors are then passed through the metric learning network to estimate a matching score. Red lines show the correspondences between the keypoints in the two 3D LiDAR scans. Different colors in the architecture represent different layers, which are explained in later sections.

a metric for matching the descriptor. Our feature learning network is based on the recent dense blocks architecture [9], and for metric learning, we use a stack of fully connected layers. Since the data used by existing learning based methods either consists of grayscale image patches or dense 3D surface patches we also generated our own training data. We extracted surface patches from LiDAR scans in the KITTI tracking benchmark [6] and obtained ground-truth correspondences by tracking keypoints using our previously proposed method [4]. Fig. 1] demonstrates the matching of keypoints using our learned feature descriptor on sparse 3D LiDAR scans.

The foremost contribution of our work is a local feature descriptor for sparse 3D LiDAR data and a metric for matching this feature descriptor. We also target relevant problems in the feature learning pipeline, i.e, extracting local surface patches and obtaining the ground-truth correspondences. To validate the performance of our feature descriptor, we evaluate the matching accuracy and compare the performance with handcrafted feature descriptors and descriptors learned with different architectures. We also present results for another experiment, where we align multiple objects based on feature correspondences. Furthermore, to highlight the difference between using a predefined and a learned metric, we present comparative results for our proposed feature descriptor using respective cases. In addition, we also show that our descriptor 
can generalize to data from different type of LiDAR sensors. We do this by repeating the alignment experiment but with data from a different sensor. We also present an ablation study to provide insight on the role of each modality we use for learning the descriptor. The training and test data, learned models and a $\mathrm{C}++$ API for using the feature descriptor with PCL is available here. 1

\section{RELATED WORK}

In this section we briefly discuss the handcrafted feature descriptors that we use for comparison with our method and an existing feature descriptor for sparse LiDAR scans. We also discuss different CNN based descriptors proposed for grayscale and dense 3D surface patches.

Several handcrafted local feature descriptors for 3D pointclouds have been proposed [2] and are currently part of the PCL library [14]. The first descriptor we compare with is the Fast Point Feature Histogram (FPFH) [15], which requires normals as input and generalizes the mean curvature around a point using a histogram. Tombari et al. [19] proposed the Signatures of Histograms of Orientations (SHOT) descriptor. Their contribution is a method for robustly estimating local reference frames and a descriptor that quantifies local surface normal information. The third image descriptor we compare with is 3D Shape Context (3DSC) [5]. Similar to other descriptors, it also requires surface normals as input and uses a spherical grid around the keypoint for counting the number of points in bins along azimuth, elevation and radial coordinates. We compare our results with these descriptors and show the advantages of using a learned feature descriptor over the handcrafted counterparts.

A feature descriptor designed for sparse 3D LiDAR scans was proposed by Serafin et al. [16]. They quantify the vertical structure in the scene with 3D lines and planes (circles) and show the efficacy of their approach by integrating these descriptors in a SLAM system. Even though their method improves the performance of the SLAM system, it might perform sub-optimally for environments that lack these vertical structures. In contrast to this, our feature descriptor is not quantifying any geometric structures in the environment and therefore can work in different environments.

With the advent of CNNs, several methods have been proposed for learning feature descriptors, but mainly for grayscale image patches. The architectures discussed in these methods consist of a network for learning the descriptor, followed by either a metric learning network for matching the descriptors or a loss layer which minimizes the distance between the descriptors using a predefined metric. The architecture proposed in MatchNet [7] consists of a Siamese network for learning the descriptor and a metric learning module. DeepCompare [20] discusses several different architectures: Siamese, pseudo-Siamese, two-channel and central-surround two-stream networks. The difference between Siamese and pseudo-Siamese is that in the latter weights are shared only for selected layers instead of every layer. In two-channel

${ }^{1}$ http://deep3d-descriptor.informatik.uni-freiburg.de architectures, the image patches are stacked as two channels instead of having a Siamese network. Central-surround twostream networks consist of four input streams, two for complete image patches and two for the central crop of the input patches. They use a loss layer minimizing Euclidean distance instead of a metric learning module and show that the central-surround two-stream architecture gives the best performance. MatchNet and DeepCompare are outperformed by recently proposed PN-Net [3]. This network architecture uses three input streams where two streams have matching image patches and the third stream is a non-matching image patch. Unlike MatcheNet, which uses softmax loss, they use SoftPN loss, a modified version of hinge loss [20].

A learning-based approach for 3D data was recently proposed by Zeng et al. [21]. Their approach called 3DMatch, targets learning descriptors for dense 3D surface patches using a Siamese network trained with contrastive $l_{2}$ loss. One of the main contributions is the proposed 3D patch representation, which uses a $3 \mathrm{D}$ voxel grid of Truncated Distance Function values for representing the 3D shape. The key difference between their work and ours is that they target dense 3D surface patches extracted after aligning multiple scans, whereas we focus on learning descriptors for single sparse 3D LiDAR scan.

We compare our proposed feature descriptor with other feature descriptors known to work with sparse 3D LiDAR data. A comparison with other learned feature descriptors is not possible because these descriptors are learned for either grayscale image patches or dense 3D surface patches. Additionally, to justify using the dense blocks based architecture for learning, we present comparative results with descriptors learned using different types of CNN architectures [7, 8]

\section{LEARNING A LOCAL FEATURE DESCRIPTOR}

Our feature learning pipeline has three main steps. First we extract keypoints and track them to obtain ground-truth correspondences. Then we extract surface patches around successfully tracked keypoints and in the last step we train the network for learning a feature descriptor as well as a metric for matching the descriptors in a unified manner.

\section{A. Generating Training Data}

A key requirement for supervised learning is labeled training data. Since labeling many correspondences by hand is a strenuous task, existing methods [7, 20, 3, 21] use 3D scene reconstruction for associating pixels corresponding to same 3D point for obtaining ground-truth correspondences. Using datasets by these methods is not possible, since our objective is to learn a feature descriptor for sparse 3D LiDAR data and the datasets made available by these methods either consists of grayscale image patches or dense 3D surface patches.

1) Ground-Truth Correspondences: To obtain groundtruth correspondences, we first select the keypoints using uniform sampling and then track those keypoints for the next five frames. For tracking, we use our previously proposed method for estimating pointwise motion [4]. Associating 


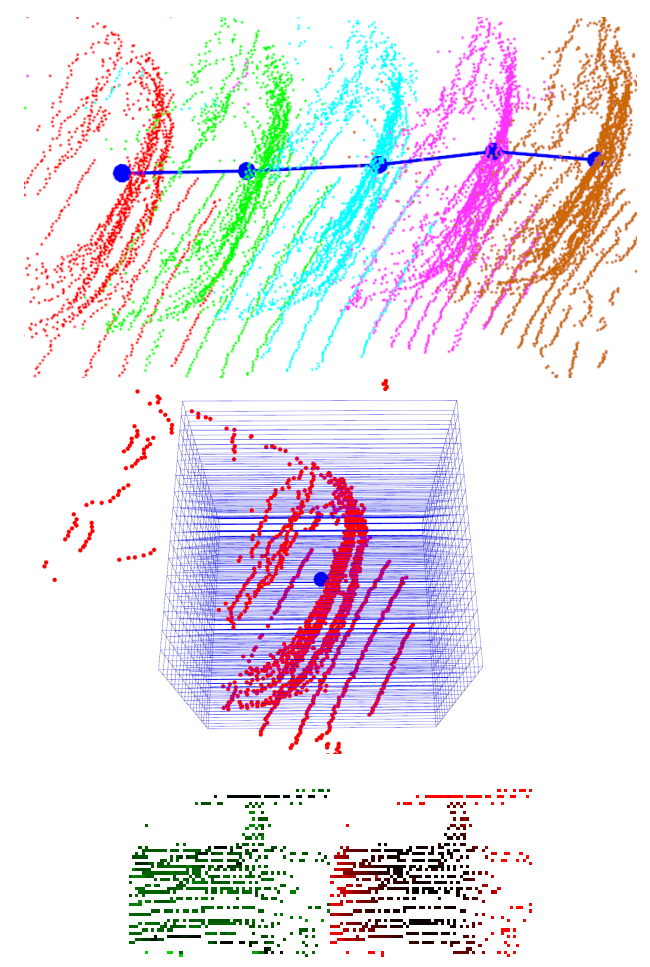

Fig. 2: The top image shows the tracked keypoint for five frames. The image in the middle shows a voxelized cube around a keypoint. The bottom image shows different channels of an extracted surface patch. Surface reflectance intensity is showed in green and depth is shown in red.

keypoints over multiple frames instead of one allows us to remove false correspondences. For keypoints that are successfully tracked over multiple frames, we extract surface patches for all five frames. The top image in Fig. 2 shows tracking of a keypoint for five frames.

We used the LiDAR scans from the KITTI tracking benchmark which consists of 20 sequences. We used the surface patches extracted from the first 10 sequences for training and the remaining 10 for testing.

2) Training Patches: Training a neural network for learning a feature descriptor requires local surface patches around keypoints. In case of 2D image data, generating patches is a straightforward task since the data is organized in a grid structure, but for unorganized sparse 3D pointclouds this task is non-trivial. In our approach, for a given keypoint, we generate a cube with predefined length that is divided into $64 \times 64 \times 1$ voxels. For every voxel we calculate the average distance w.r.t the keypoint and the average surface reflectance intensity values for the points inside the voxel. and store the $3 \mathrm{D}$ voxel as a two channel image patch $(64 \times 64 \times 2)$. The first modality (depth) aims at capturing the geometry and the second (intensity) captures surface reflectance properties. The local extent in which information has to be captured around a keypoint is defined by the length of the cube. Fig. 2 illustrates this process, where the middle image shows the voxel structure around a keypoint and the bottom image shows the modalities we use.

\section{B. Network Architecture}

Fig. 3(a) shows the network architecture that we use in our approach. The architecture consists of a two-stream Siamese network for learning the features, followed by a metric learning network. Each stream of the Siamese network consists of two convolution layers followed by two dense blocks and a bottleneck layer. Dense blocks based networks [9] have been recently shown to improve the state-of-the-art for different tasks. According to the authors [9], the improvement in performance is mainly attributed to the better exploitation of feature re-use capability which allows efficient flow of information resulting in better feature representation.

Each dense block consists of two layers, where each layer is a composite function consisting of batch-normalization, Rectified Linear Unit (ReLU), and a convolution operation. The bottleneck layer is a convolution layer and output of it is the learned feature descriptor.

In the metric learning module, we have 5 fully connected layers $(f c)$ where every $f c$ layer, except the last one $\left(f_{c} 4\right)$, is followed by a ReLU. The input to the metric learning module is the concatenation of the output of each stream of the Siamese network. The number of feature maps for every $f_{c}$ layer is shown in Fig. 3(b).

Our unified feature and metric learning problem can be seen as a binary classification problem, where a pair of input patches have to be classified as matching or non-matching. Our training set is $\mathcal{T}=\left\{\left(X_{n}^{1}, X_{n}^{2}, Y_{n}\right), n=1, \ldots, N\right\}$, where $X_{n}^{1}$ and $X_{n}^{2}$ are two sets of surface patches and $Y_{n}=$ $\left\{y_{k} \in\{1,0\}, k=1, \ldots, N\right\}$ is the corresponding ground truth labels. The activation function for our learning model is defined as $f\left(x_{k}^{1}, x_{k}^{2}, \theta\right)$, where $\theta$ are the parameters of our model and $x_{k}^{1} \in X_{n}^{1}$ and $x_{k}^{2} \in X_{n}^{2}$ is a pair of surface patches. The network learns the weights $\theta$ by minimizing the cross-entropy (softmax) loss in Eq. 1. over all patch pairs as shown in Eq. 2. To avoid overfitting we add a term for $l_{2}$ regularization to the loss function in Eq. 2

$$
\begin{gathered}
\mathcal{L}(p, q)=-\sum_{c \in\{0,1\}} p_{c} \log q_{c} \\
\theta^{*}=\underset{\theta}{\operatorname{argmin}} \frac{1}{N} \sum_{k=1}^{N} \mathcal{L}\left(y_{k}, f\left(x_{k}^{1}, x_{k}^{2}, \theta\right)\right)+\frac{\eta}{2}\|\theta\|_{2}^{2}
\end{gathered}
$$

An alternative to metric learning is to use a loss layer directly after the feature learning. Most commonly used loss layers are contrastive loss [21] and hinge embedding loss [17]. Both of these layers try to minimize the Euclidean distance between matching descriptor pairs and simultaneously increase the margin between non-matching pairs. In section $[\mathrm{IV}$ we present a comparison between our proposed network for unified learning and our modified feature learning network trained with hinge embedding loss. In the modified version we replace the last convolution layer (Fig. 3(b) with a set of fully connected layers, where the output of the last layer is the feature descriptor. 


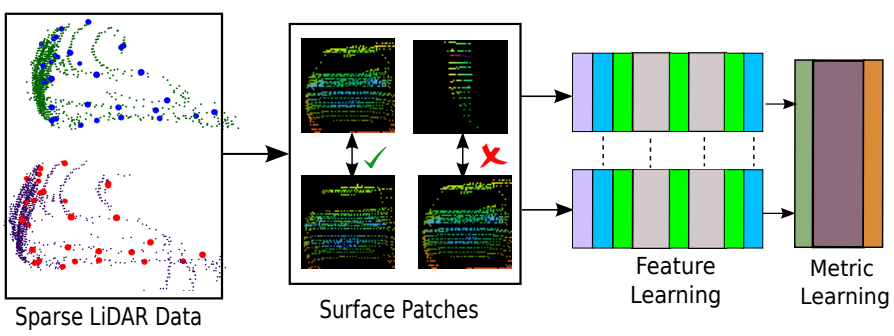

(a)

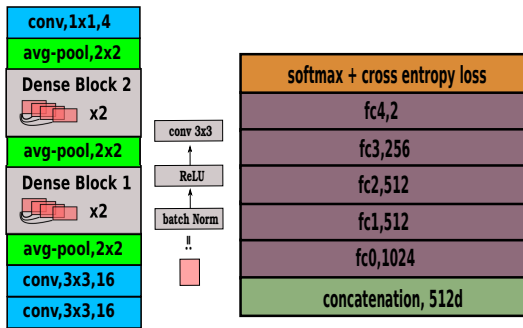

(b)

Fig. 3: (a) An overview of our feature learning method. The local information around the keypoints from sparse LiDAR scans is converted into surface patches. Input to the feature learning network is a pair of matching and a pair of non-matching surface patches and input to the metric learning network is the learned feature descriptors. In (b) we show the architecture for each module. Feature learning module is a Siamese network, where filters in each layer share weights and the metric learning network contains a stack of fully connected layers.

\section{Training}

Our complete network architecture is implemented in TensorFlow [1]. Using our patch generation method, we generated 58,710 surface patches for training. The input to the network is a batch of surface patch pairs. Each batch consists of an equal number of matching and non-matching surface patches as shown in Fig. 3(a) With these surface patches, we estimated 117,400 positive and 704,400 negative pairs. Since the input to the network always consists of a negative and positive combination, our effective training set consists of 704,400 samples.

We train our network with a batch size of 32 and use the Adam optimizer [10] with a learning rate of $1 e^{-4}$. The parameter $\eta$ for $l_{2}$-norm regularization was fixed to $5 e^{-4}$. The growth rate for dense blocks is 4 . The network was trained for 5 epochs and the complete training process required around 2 hours on an NVIDIA GeForce GTX 980 graphics card.

\section{Results}

To evaluate our method, we perform multiple experiments. We first evaluate the matching accuracy for various descriptors. We then report the average alignment errors for objects scanned using Velodyne HDL-64E and HDL-32E LiDAR scanners. We also report computation times for calculating and matching the different descriptors. For all experiments we compare the proposed feature descriptors with other feature descriptors. Among handcrafted descriptors, we compare with SHOT [19], FPFH [15], and 3DSC [5]. To justify the usage of dense blocks for our task, we present results for feature descriptors learned with the following two different network architectures [7, 8].

1) The first architecture [7] was proposed to learn a feature descriptor and a metric for grayscale image patches. Their feature learning architecture consists of blocks of convolution layers and ReLUs separated by maxpooling layers and they use a stack of fully connected layers for metric learning. This feature learning architecture is similar to the initially proposed CNN architectures for instance VGG [18]. Other methods [21, 20]

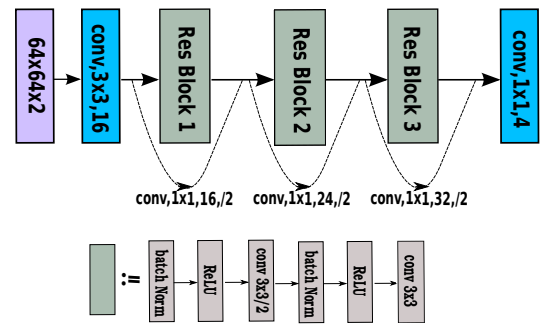

Fig. 4: Architecture for ResNet-8

proposed for learning feature descriptors have also used similar architectures. The reason we chose the architecture from MatchNet is because they have similar input patch size as ours $(64 \times 64)$ and also use metric learning.

2) The second architecture we compare with consists of residual blocks [8]. Lately, residual blocks based architectures have also shown to perform well for a variety of tasks [8, 12]. We use a ResNet-8 [12] for feature learning and keep the metric learning architecture unchanged. Fig. 4 shows the ResNet- 8 architecture we used.

Furthermore, we also compare with our feature learning network trained with the hinge embedding (H.E.) loss. The first three comparisons present the advantages of learned feature descriptors over the handcrafted descriptors. The next two comparisons highlight the benefits of using dense blocks while the last one compares the performance of a learned metric with a predefined metric.

\section{A. Matching Accuracy}

The goal of this experiment is to test the performance of different descriptors on the pairs of surface patches from our testing set. We use 100,000 samples, half of them are matching and the other half are non-matching. For every case we plot a receiver operating characteristic (ROC) curve and report false-positive rate at 95\% recall (FPR95). The ROC curves are shown in Fig. 5 and the FPR95 is reported in Table [ The curve is plotted for various matching thresholds, which in case of metric learning is the softmax score. For the handcrafted descriptors and the descriptor learned using 


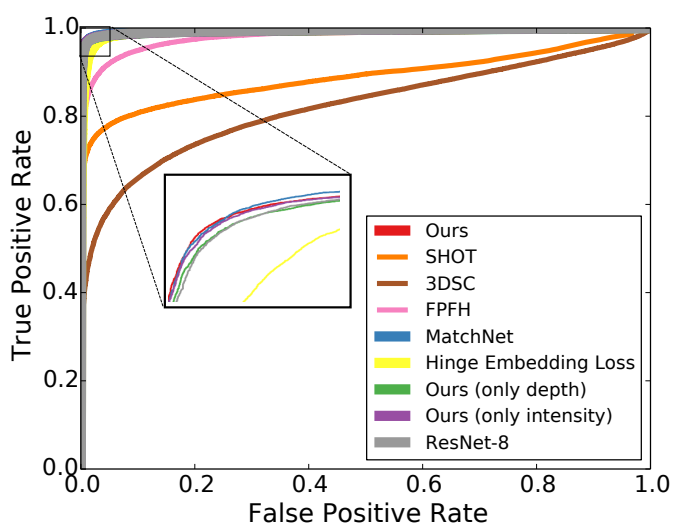

Fig. 5: ROC curves for different feature descriptors. Performance of our proposed feature descriptor is comparable to MatchNet. Using H.E. loss instead of metric learning leads to a decrease in performance, whereas all handcrafted descriptors underperform in comparison to the learned descriptors.

H.E. loss, the Euclidean distance between descriptors is used as matching threshold.

The error for our feature descriptor is the lowest and it outperforms the handcrafted descriptors by a significant margin. The next best performing feature descriptors is learned using the architecture of MatchNet, followed by the ResNet-8 architecture. These results justify learning a feature descriptor using our proposed architecture.

The error increases when our feature learning network is trained with H.E. loss (yellow curve in Fig. 55), demonstrating the importance of metric learning. The increase in performance due to metric learning comes at the cost of an increase in matching time due to computationally expensive forward pass through the metric learning network. We discuss the matching time for different descriptors in next section.

TABLE I: FPR95 Error

\begin{tabular}{|c|c|c|}
\hline Method & Feature Size & Error(\%) \\
\hline SHOT \19] & 352 & 82.56 \\
FPFH [15] & 33 & 10.26 \\
3DSC [5] & 1980 & 89.16 \\
MatchNet [7] & 4096 & 0.45 \\
ResNet-8 & 256 & 0.60 \\
Ours (H.E. Loss) & 256 & 1.94 \\
Ours (depth + intensity) & 256 & $\mathbf{0 . 4 2}$ \\
Ours (only depth) & 256 & 0.46 \\
Ours (only intensity) & 256 & 0.53 \\
\hline
\end{tabular}

\section{B. Alignment}

Many methods for surface or scan registration require coarse initial alignment, especially when data is collected at a low rate (typically $10 \mathrm{~Hz}$ for LiDAR) and the assumption that nearest neighbor points are corresponding does not hold. In this experiment we align multiple objects scanned using two different LiDAR scanners. In Table III, we report the number of objects used in the experiment, number of points belonging to each object, number of keypoints and the translational and rotational alignment error of our method.
TABLE II: Alignment error of our method for the individual objects

\begin{tabular}{|c|c|c|c|c|}
\hline Object ID & Points & Keypoints & $t_{e}(\mathrm{~m})$ & $r_{e}(\mathrm{rad})$ \\
\hline \multicolumn{5}{|c|}{ Scans from Velodyne HDL-64E } \\
\hline 0 & 1369 & 483 & 0.28 & 0.04 \\
1 & 493 & 285 & 0.54 & 0.04 \\
2 & 787 & 332 & 0.47 & 0.04 \\
3 & 250 & 186 & 0.52 & 0.02 \\
4 & 1320 & 383 & 1.55 & 0.24 \\
5 & 970 & 394 & 1.06 & 0.13 \\
6 & 228 & 129 & 1.48 & 0.10 \\
7 & 199 & 154 & 1.10 & 0.06 \\
8 & 580 & 395 & 0.73 & 0.02 \\
9 & 564 & 427 & 0.08 & 0.02 \\
10 & 316 & 233 & 1.22 & 0.04 \\
11 & 1517 & 908 & 0.23 & 0.01 \\
\hline \multicolumn{5}{|c|}{ Scans from Velodyne HDL-32E } \\
\hline 0 & 313 & 230 & 0.88 & 0.07 \\
1 & 3271 & 1099 & 0.46 & 0.13 \\
2 & 3741 & 1239 & 0.49 & 0.13 \\
3 & 319 & 239 & 0.22 & 0.14 \\
\hline
\end{tabular}

TABLE III: Average alignment errors for HDL-64E scans

\begin{tabular}{|c|c|c|c|c|c|}
\hline \multirow{2}{*}{ Method } & \multicolumn{2}{|c|}{ raw } & \multicolumn{2}{c|}{ RANSAC } & \multirow{2}{*}{$\mathrm{t}(\mathrm{s})$} \\
\cline { 2 - 5 } & $t_{e}(\mathrm{~m})$ & $r_{e}(\mathrm{rad})$ & $t_{e}(\mathrm{~m})$ & $r_{e}(\mathrm{rad})$ & \\
\hline SHOT [19] & $1.38 \pm 1.06$ & $0.13 \pm 0.11$ & $0.76 \pm 0.56$ & $0.10 \pm 0.07$ & 0.05 \\
FPFH [15] & $3.37 \pm 5.40$ & $0.33 \pm 0.54$ & $1.30 \pm 0.89$ & $0.14 \pm 0.11$ & $\mathbf{0 . 0 0 4}$ \\
3DSC [5] & $2.75 \pm 2.58$ & $0.34 \pm 0.38$ & $0.81 \pm 0.37$ & $0.08 \pm 0.04$ & 0.57 \\
MatchNet [7 & $0.88 \pm 0.47$ & $0.08 \pm 0.06$ & $0.93 \pm 0.77$ & $0.07 \pm 0.05$ & 14.78 \\
ResNet-8 & $0.95 \pm 0.48$ & $0.077 \pm 0.067$ & $0.83 \pm 0.71$ & $0.08 \pm 0.05$ & 1.03 \\
H.E. loss & $\mathbf{0 . 7 6} \pm \mathbf{0 . 3 4}$ & $0.076 \pm 0.062$ & $\mathbf{0 . 5 2} \pm \mathbf{0 . 2 7}$ & $\mathbf{0 . 0 5} \pm \mathbf{0 . 0 1}$ & 0.18 \\
Ours & $0.77 \pm 0.47$ & $\mathbf{0 . 0 7 1} \pm \mathbf{0 . 0 6 1}$ & $0.57 \pm 0.28$ & $0.06 \pm 0.02$ & 0.92 \\
\hline
\end{tabular}

TABLE IV: Average alignment errors for HDL-32E scans

\begin{tabular}{|c|c|c|c|c|}
\hline \multirow{2}{*}{ Method } & \multicolumn{2}{|c|}{ raw } & \multicolumn{2}{c|}{ RANSAC } \\
\cline { 2 - 5 } & $t_{e}(\mathrm{~m})$ & $r_{e}(\mathrm{rad})$ & $t_{e}(\mathrm{~m})$ & $r_{e}(\mathrm{rad})$ \\
\hline SHOT [19] & $0.57 \pm 0.29$ & $0.11 \pm 0.04$ & $0.46 \pm 0.54$ & $0.07 \pm 0.06$ \\
FPFH [15] & $1.32 \pm 1.08$ & $0.22 \pm 0.10$ & $2.07 \pm 2.20$ & $0.14 \pm 0.25$ \\
3DSC [5] & $2.48 \pm 3.46$ & $0.28 \pm 0.27$ & $0.73 \pm 0.77$ & $0.06 \pm 0.03$ \\
MatchNet [7] & $0.97 \pm 1.01$ & $0.13 \pm 0.08$ & $0.88 \pm 1.24$ & $0.14 \pm 0.11$ \\
ResNet-8 & $0.81 \pm 0.33$ & $0.14 \pm 0.06$ & $0.60 \pm 0.56$ & $0.07 \pm 0.04$ \\
H.E. loss & $0.65 \pm 0.14$ & $\mathbf{0 . 0 7} \pm \mathbf{0 . 0 6}$ & $0.58 \pm 0.55$ & $0.11 \pm 0.04$ \\
Ours & $\mathbf{0 . 5 1} \pm \mathbf{0 . 2 3}$ & $0.12 \pm 0.02$ & $\mathbf{0 . 4 1} \pm \mathbf{0 . 3 9}$ & $\mathbf{0 . 0 5} \pm \mathbf{0 . 0 5}$ \\
\hline
\end{tabular}

1) LiDAR Scans from Velodyne HDL-64E: In this experiment we align 12 static objects extracted from consecutive LiDAR scans from the KITTI tracking benchmark. Since all objects are static, the ground-truth motion is the inverse sensor motion, which is provided by the benchmark. In Fig.6 we show example results, where the image on the left shows the misaligned objects and the corresponding points, whereas the right image illustrates the aligned pointclouds. As before, we use uniform sampling for selecting keypoints. In Table III. we report the average translational error $t_{e}$ and rotational error $r_{e}$ for the motion estimated using the raw point correspondences and correspondences filtered with RANSAC. The motion estimated using raw correspondences reflects more clearly on the feature matching accuracy in comparison to filtered correspondences. The alignment error reported for our method using raw correspondences is average of the errors reported in Table II While our feature descriptors with learned metric and hinge embedding loss perform similar, they outperform the other descriptors by significant margin.

The rightmost column in Table III reports the feature 

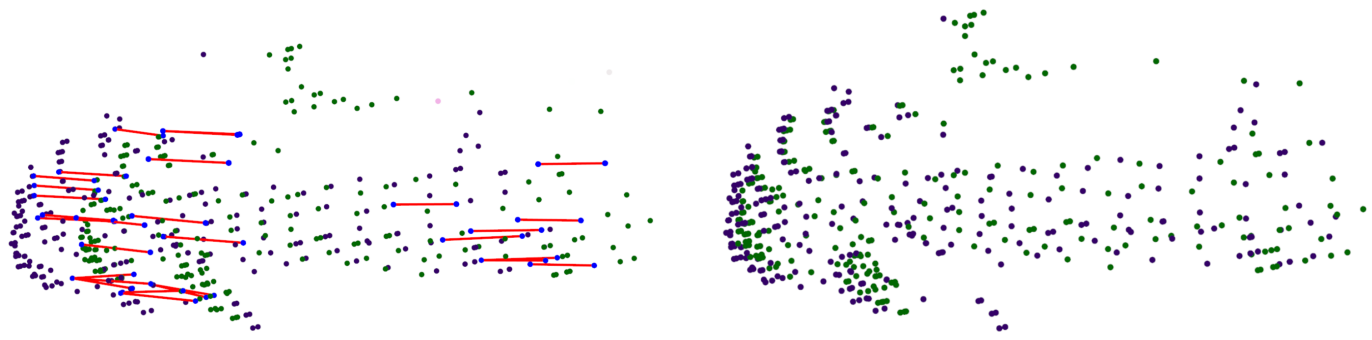

Fig. 6: An illustration of the alignment experiment. The image on the left shows the sparse misaligned objects and the correspondences estimated by matching our feature descriptor. The image on the right shows the aligned pointclouds.

matching time. The FPFH descriptor takes minimum time for matching but has the largest alignment error. All the handcrafted descriptors can be matched quickly using KD-trees in comparison to feature descriptors learned using a metric and therefore they have the lowest matching time. In this experiment our feature descriptor learned using Euclidean distance performs most favorably considering both alignment error and matching time. Among the feature descriptors learned using the metric, the time for MatchNet is the largest. This increase in time is mainly attributed to the large descriptor size (4096 vs. 256) which results in twice as much as parameters in the metric learning network in comparison to other learned descriptors. The matching time of ResNet-8 is similar to ours, since the architecture for metric learning and the feature descriptor size is the same in both cases.

2) LiDAR Scans from Velodyne HDL-32E: We repeat the alignment experiment like above but with the data collected from a 32 beam LiDAR scanner. Like before we align static objects and use the sensor pose from the SLAM solution [11] as ground-truth motion. The purpose of this experiment is to show that our feature descriptor is not overfitting to data collected from a single sensor but can generalize to data collected from different sensors. Even though both sensors provide the same modalities, the data from Velodyne HDL$32 \mathrm{E}$ is often sparser in comparison to data from Velodyne HDL-64E and also the has different measurement noise. Table IV] shows the alignment error for different cases and our feature descriptor learned with metric outperforms the other descriptors. This experiment demonstrates that our proposed feature descriptor is capable of generalizing on data from different sensors.

TABLE V: Per feature computation time (in ms) for various neighborhood radii.

\begin{tabular}{|c|c|l|l|l|l|c|}
\hline \multirow{2}{*}{ Method } & \multirow{2}{*}{ Processor } & \multicolumn{5}{|c|}{ Neighborhood radius $(\mathrm{m})$} \\
\cline { 3 - 7 } & & \multicolumn{1}{|c|}{0.4} & 0.8 & \multicolumn{1}{|c|}{1.6} & 3.2 & 6.4 \\
\hline SHOT \19] & MC & 0.092 & $\mathbf{0 . 0 9 6}$ & $\mathbf{0 . 1 1 9}$ & 0.284 & 0.787 \\
FPFH [15] & MC & $\mathbf{0 . 0 9 0}$ & 0.282 & 0.961 & 3.01 & 10.23 \\
3DSC [5] & $\mathrm{C}$ & 0.148 & 0.440 & 3.21 & 31.19 & 344.10 \\
MatchNet [7] & MC+ & $0.036+$ & $0.037+$ & $0.047+$ & $0.075+$ & $0.219+$ \\
& $\mathrm{G}$ & 0.139 & 0.139 & 0.139 & 0.139 & 0.139 \\
ResNet-8 & MC+ & $0.036+$ & $0.037+$ & $0.047+$ & $0.075+$ & $0.219+$ \\
& $\mathrm{G}$ & 0.151 & 0.151 & 0.151 & 0.151 & 0.151 \\
H.E. loss & $\mathrm{MC}+$ & $0.036+$ & $0.037+$ & $0.047+$ & $\mathbf{0 . 0 7 5 +}$ & $\mathbf{0 . 2 1 9 +}$ \\
& $\mathrm{G}$ & 0.132 & 0.132 & 0.132 & $\mathbf{0 . 1 3 2}$ & $\mathbf{0 . 1 3 2}$ \\
Ours & $\mathrm{MC}+$ & $0.036+$ & $0.037+$ & $0.047+$ & $0.075+$ & $0.219+$ \\
& $\mathrm{G}$ & 0.133 & 0.133 & 0.133 & 0.133 & 0.133 \\
\hline
\end{tabular}

TABLE VI: Computation time (in seconds) for various sampling radii.

\begin{tabular}{|c|c|c|c|c|c|c|c|}
\hline \multirow{2}{*}{ Method } & \multicolumn{7}{|c|}{ Sampling radius $(\mathrm{m})$} \\
\cline { 2 - 8 } & 3.2 & 1.6 & 0.8 & 0.4 & 0.2 & 0.1 & 0.05 \\
\hline SHOT [19] & 0.97 & 0.97 & 1.36 & 2.64 & 6.45 & 12.47 & 24.83 \\
FPFH [15] & 27.97 & 28.30 & 27.86 & 31.87 & 40.74 & 55.79 & 85.13 \\
3DSC [5] & 8.97 & 35.07 & 99.49 & 355.58 & 1111.65 & 3073.30 & 6179.10 \\
MatchNet [7] & $0.03+$ & $0.11+$ & $\mathbf{0 . 2 8}+$ & $0.81+$ & $3.72+$ & $4.40+$ & $9.17+$ \\
& 0.22 & 0.33 & $\mathbf{0 . 6 7}$ & 1.68 & 3.29 & 6.25 & 8.26 \\
ResNet-8 & $\mathbf{0 . 0 3 +}$ & $\mathbf{0 . 1 1 +}$ & $0.28+$ & $0.81+$ & $3.72+$ & $4.40+$ & $9.17+$ \\
& $\mathbf{0 . 2 0}$ & $\mathbf{0 . 1 6}$ & 0.70 & 1.68 & 3.61 & 6.75 & 9.84 \\
H.E. loss & $0.03+$ & $0.11+$ & $0.28+$ & $\mathbf{0 . 8 1 +}$ & $\mathbf{3 . 7 2 +}$ & $\mathbf{4 . 4 0 +}$ & $\mathbf{9 . 1 7 +}$ \\
& 0.33 & 0.42 & 0.70 & $\mathbf{1 . 4 7}$ & $\mathbf{3 . 0 1}$ & $\mathbf{5 . 4 3}$ & $\mathbf{7 . 8 0}$ \\
Ours & $0.03+$ & $0.11+$ & $0.28+$ & $0.81+$ & $3.72+$ & $4.40+$ & $9.17+$ \\
& 0.34 & 0.43 & 0.71 & 1.49 & 3.05 & 5.52 & 7.90 \\
\hline
\end{tabular}

\section{Computation Time}

In Table $\mathrm{V}$ and $\mathrm{VI}$, we report the computation time for estimating feature descriptors for different neighborhood radii and sampling radii. In the first case, we estimate descriptors for the same number of points (sampling radius of $0.4 \mathrm{~m}$ ) but for different neighborhood radii. This evaluation highlights per feature calculation time, which only depends on the input neighborhood radius. In the second case, we use a fixed neighborhood radius of $3.2 \mathrm{~m}$ while varying the sampling radius. This evaluation focuses on changes in computation time with the increase in number of keypoints. For each case we report the processor details, i.e whether it is a multi-core CPU (MC), single core CPU (C) or GPU (G). For learning methods, we separately report the time required for estimating the surface patches and the time required for estimating the feature descriptors.

For small neighborhood radii, handcrafted descriptors have low computation time because different per-point operation like estimating normals and estimating descriptor among other different operations, are performed efficiently for smaller radii (fewer points). While increasing these radii (more points), these operations are not as efficient as before even with usage of KD-Trees. In case of the learned feature descriptors, the change in neighborhood radii only affects the patch computation time and not the feature estimation time. For patch computation the voxelization of the neighborhood is independent of the number of points in the neighborhood. The only operation dependent on number of points is calculating the average in depth and intensity values. Among the learned descriptors, our feature descriptor requires least computation time in comparison to other architectures be- 
cause our feature learning network has the least number of parameters as well.

With the decrease in sampling radius, the number of keypoints increases. The reported time is the combined computation time for all the keypoints. In this case, the better performance of learned feature descriptors is mainly attributed to the proper utilization of parallel processing capabilities of GPUs in comparison to the multi-core implementation of handcrafted descriptors on CPUs. In comparison to other learned descriptors, the performance of our feature learning network scales better with the increase in number of keypoints. Since our network is smaller than others, it allows us to process larger batches of data in parallel.

\section{Ablation Study}

To better understand the contribution of each of the modality, we trained two separate networks with single channel input (depth and intensity). Table $\mathrm{I}$ shows the FPR95 error for both cases. The performance of the feature descriptor learned using depth is better than the feature descriptor learned using surface intensity values. Since the surface reflectance values often depend on the angle at which laser beam hits the surface, they are not as stable as the depth values, especially in the case when the sensor is moving. The error for both of these cases is higher than the feature descriptor learned using both modalities and therefore using them together helps in learning a more discriminative feature descriptor.

\section{CONCLUSions}

In this work, we propose a local feature descriptor for 3D LiDAR scans and a metric for matching the descriptors. We use an architecture based on dense blocks for feature learning and show how our architecture learns more discriminative feature descriptors in comparison to descriptors learned using other common architectures. We report results on matching accuracy and the alignment error. For both cases our learned descriptor outperforms handcrafted descriptors by a significant margin. We also report results for data collected from a different sensor and demonstrate how our descriptor can generalize to different sources of data. We compare the performance of a descriptor learned using a predefined metric and learned with a metric and show that using former is suitable when faster matching time is necessary. Additionally, we also present an ablation study to understand the importance of depth and intensity modalities and show that using them together enables learning of a more discriminative feature descriptor.

\section{REFERENCES}

[1] Martín Abadi, Ashish Agarwal, Paul Barham, Eugene Brevdo, Zhifeng Chen, Craig Citro, Greg S Corrado, Andy Davis, Jeffrey Dean, Matthieu Devin, et al. Tensorflow: Large-scale machine learning on heterogeneous distributed systems. arXiv preprint arXiv:1603.04467, 2016.

[2] Luis A Alexandre. 3d descriptors for object and category recognition: a comparative evaluation. In Workshop on Color-Depth Camera Fusion in Robotics at the IEEE/RSJ International Conference on Intelligent Robots and Systems (IROS), Vilamoura, Portugal, volume 1, page 7, 2012.
[3] Vassileios Balntas, Edward Johns, Lilian Tang, and Krystian Mikolajczyk. Pn-net: conjoined triple deep network for learning local image descriptors. arXiv preprint arXiv:1601.05030, 2016.

[4] Ayush Dewan, Tim Caselitz, Gian Diego Tipaldi, and Wolfram Burgard. Rigid scene flow for $3 \mathrm{~d}$ lidar scans. In IEEE/RSJ International Conference on Intelligent Robots and Systems (IROS), 2016.

[5] Andrea Frome, Daniel Huber, Ravi Kolluri, Thomas Bülow, and Jitendra Malik. Recognizing objects in range data using regional point descriptors. Computer vision-ECCV 2004, pages 224-237, 2004.

[6] Andreas Geiger, Philip Lenz, and Raquel Urtasun. Are we ready for autonomous driving? the kitti vision benchmark suite. In IEEE Conference on Computer Vision and Pattern Recognition (CVPR), 2012.

[7] Xufeng Han, Thomas Leung, Yangqing Jia, Rahul Sukthankar, and Alexander C Berg. Matchnet: Unifying feature and metric learning for patch-based matching. In Proceedings of the IEEE Conference on Computer Vision and Pattern Recognition, pages 3279-3286, 2015.

[8] Kaiming He, Xiangyu Zhang, Shaoqing Ren, and Jian Sun. Deep residual learning for image recognition. In Proceedings of the IEEE conference on computer vision and pattern recognition, pages 770 $778,2016$.

[9] Gao Huang, Zhuang Liu, Laurens van der Maaten, and Kilian Q Weinberger. Densely connected convolutional networks. In Proceedings of the IEEE Conference on Computer Vision and Pattern Recognition, 2017.

[10] Diederik Kingma and Jimmy Ba. Adam: A method for stochastic optimization. arXiv preprint arXiv:1412.6980, 2014.

[11] Rainer Kümmerle, Michael Ruhnke, Bastian Steder, Cyrill Stachniss, and Wolfram Burgard. Autonomous robot navigation in highly populated pedestrian zones. Journal of Field Robotics, 32(4):565-589, 2015.

[12] Antonio Loquercio, Ana Isabel Maqueda, Carlos R. Del Blanco, and Davide Scaramuzza. Dronet: Learning to fly by driving. IEEE Robotics and Automation Letters, 2018. doi: 10.1109//ra.2018.2795643.

[13] Tayyab Naseer, Luciano Spinello, Wolfram Burgard, and Cyrill Stachniss. Robust visual robot localization across seasons using network flows. In Proc. of the AAAI Conference on Artificial Intelligence, 2014

[14] Radu Bogdan Rusu and Steve Cousins. 3d is here: Point cloud library (pcl). In Robotics and automation (ICRA), 2011 IEEE International Conference on, pages 1-4. IEEE, 2011.

[15] Radu Bogdan Rusu, Nico Blodow, and Michael Beetz. Fast point feature histograms (fpfh) for $3 \mathrm{~d}$ registration. In Robotics and Automation, 2009. ICRA'09. IEEE International Conference on, pages 3212-3217. IEEE, 2009.

[16] Jacopo Serafin, Edwin Olson, and Giorgio Grisetti. Fast and robust 3d feature extraction from sparse point clouds. In Intelligent Robots and Systems (IROS), 2016 IEEE/RSJ International Conference on, pages 4105-4112. IEEE, 2016.

[17] Edgar Simo-Serra, Eduard Trulls, Luis Ferraz, Iasonas Kokkinos, Pascal Fua, and Francesc Moreno-Noguer. Discriminative learning of deep convolutional feature point descriptors. In Proceedings of the IEEE International Conference on Computer Vision, pages 118-126, 2015.

[18] K. Simonyan and A. Zisserman. Very deep convolutional networks for large-scale image recognition. CoRR, abs/1409.1556, 2014.

[19] Federico Tombari, Samuele Salti, and Luigi Di Stefano. Unique signatures of histograms for local surface description. In European Conference on Computer Vision (ECCV). Springer, 2010.

[20] Sergey Zagoruyko and Nikos Komodakis. Learning to compare image patches via convolutional neural networks. In Proceedings of the IEEE Conference on Computer Vision and Pattern Recognition, pages 43534361, 2015.

[21] Andy Zeng, Shuran Song, Matthias Nießner, Matthew Fisher, Jianxiong Xiao, and Thomas Funkhouser. 3dmatch: Learning local geometric descriptors from rgb-d reconstructions. In CVPR, 2017. 